
\title{
Inspiratory muscle load and capacity in chronic heart failure
}

\author{
N Hart, M T Kearney, N B Pride, M Green, F Lofaso, A M Shah, J Moxham, M I Polkey
}

Thorax 2004;59:477-482. doi: 10.1136/thx.2003.007724

See end of article for authors' affiliations ....................

Correspondence to: Dr N Hart, Respiratory Muscle Laboratory, Royal Brompton Hospital, London SW3 6NP, UK: drnhart@aol.com

Received 28 March 2003 Accepted 13 November 2003

\begin{abstract}
Background: Although breathlessness is common in chronic heart failure (CHF), the role of inspiratory muscle dysfunction remains unclear. We hypothesised that inspiratory muscle endurance, expressed as a function of endurance time (Tlim) adjusted for inspiratory muscle load and inspiratory muscle capacity, would be reduced in CHF.

Methods: Endurance was measured in 10 healthy controls and 10 patients with CHF using threshold loading at $40 \%$ maximal oesophageal pressure (Poes $\max$ ). Oesophageal pressure-time product (PTPoes

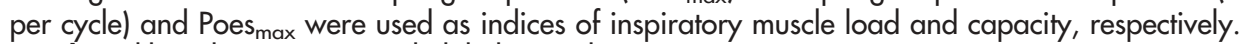

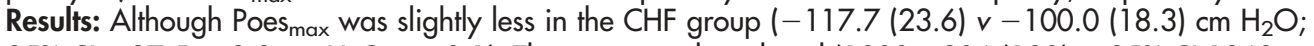
$95 \% \mathrm{Cl}-37.5$ to $2.2 \mathrm{~cm} \mathrm{H}_{2} \mathrm{O}, \mathrm{p}=0.1$ ), Tlim was greatly reduced (1800 v 306 (190) s; $95 \% \mathrm{Cl} 1368$ to $1620 \mathrm{~s}, \mathrm{p}<0.0001)$ and the observed PTPoes per cycle/Poes $\max$ was increased $(0.13(0.05) \vee 0.21$ (0.04); $95 \% \mathrm{Cl}-0.11$ to $-0.03, \mathrm{p}=0.001)$. Most of this increased inspiratory muscle load was due to a maladaptive breathing pattern, with a reduction in expiratory time $(3.0(5.8)$ v $1.1(0.3) \mathrm{s} ; 95 \% \mathrm{Cl} 0.3$ to $3.5 \mathrm{~s}, \mathrm{p}=0.03$ ) accompanied by an increased inspiratory time relative to total respiratory cycle (Ti/Ttot) $(0.43(0.14) \vee 0.62(0.07) ; 95 \% \mathrm{Cl}-0.3$ to $-0.1, p=0.001)$. However, log Tlim, which incorporates the higher inspiratory muscle load to capacity ratio caused by this altered breathing pattern, was $\geqslant 85 \%$ predicted in seven of 10 patients.

Conclusions: Although a marked reduction in endurance time was observed in CHF, much of this reduction was explained by the increased inspiratory muscle load to capacity ratio, suggesting that the major contributor to task failure was a maladaptive breathing pattern rather than impaired inspiratory muscle endurance.
\end{abstract}

$\mathrm{B}$ reathlessness is a common symptom in chronic heart failure (CHF). ${ }^{12}$ Although an imbalance between the pressure generated by the inspiratory muscles and the maximal pressure achievable contributes to dyspnoea, ${ }^{3}$ the role of impaired inspiratory muscle endurance remains unclear. Early studies ${ }^{4-6}$ suggested that patients with heart failure have marked inspiratory muscle weakness but, using more detailed invasive techniques, later studies have shown that inspiratory muscle strength is only modestly reduced..$^{7-9}$ Nevertheless, there is evidence of excessive loading of the inspiratory muscles during exhaustive exercise, ${ }^{710}$ but without overt diaphragm fatigue. ${ }^{71}$ These observations suggest that, in patients with CHF, an imbalance may exist between the capacity of the inspiratory muscle pump and its ability to sustain activity against inspiratory loads rather than straightforward weakness of the inspiratory muscles.

Recent data from our laboratory have shown that the endurance of the inspiratory muscles, assessed using a technique of constant level negative pressure threshold loading, is a function of endurance time (Tlim) and the ratio of the inspiratory muscle load to inspiratory muscle capacity. ${ }^{12}{ }^{13}$ Compared with previous methods, ${ }^{14}{ }^{15}$ this technique directly assesses the inspiratory muscle load to capacity ratio and is novel because it allows for, and permits the evaluation of, patient initiated changes in breathing pattern that occur in response to high inspiratory loads. ${ }^{16}$ Thus, it has been shown previously that a reduction in Tlim does not necessarily imply significant impairment of inspiratory muscle endurance, but may indicate a particular breathing strategy response to loaded breathing. ${ }^{12}{ }^{13}$ In the current study we hypothesised that there would be an overall reduction in inspiratory muscle endurance in patients with CHF. However, the main aim was to quantify the reduction in inspiratory muscle endurance and to examine the magnitude of the inspiratory muscle load to capacity ratio and assess its effects on Tlim.

\section{METHODS}

The protocol was approved by the local ethics committee. In line with the previous studies of inspiratory muscle endurance in patients with pulmonary disease, ${ }^{12}{ }^{13} 10$ patients with CHF (nine men) recruited from our cardiology department and 10 healthy controls (nine men) were studied. The healthy volunteers were taken from hospital staff and their relatives. Patients with neuromuscular and pulmonary disease were excluded.

\section{Cardiac and pulmonary function testing}

$\mathrm{CHF}$ was defined as stable symptomatic left ventricular dysfunction with a left ventricular ejection fraction (LVEF) $<45 \% .{ }^{17}$ LVEF and left ventricular end diastolic dimensions (LVEDD) were measured by two dimensional and M mode echocardiography performed by a physician blinded to the results of the study. Pulmonary function tests were performed according to standard guidelines ${ }^{18}$ and expressed as a percentage of published values. ${ }^{19}$

\footnotetext{
Abbreviations: $\mathrm{BMI}$, body mass index; $\mathrm{CHF}$, chronic heart failure; CLdyn, dynamic lung compliance; $\mathrm{FEV}_{1}$, forced expiratory volume in 1 second; FRC, functional residual capacity; FVC, forced vital capacity; LVEDD, left ventricular end diastolic dimension; LVEF, left ventricular ejection fraction; NYHA, New York Heart Association functional class; Poes, oesophageal pressure; Poes exp, peak expiratory oesophageal pressure; Poes $\max _{\text {, }}$ maximum negative oesophageal pressure during an inspiratory manoeuvre; Poes peak $_{\text {/TTPoes }}$ peak, inspiratory muscle contraction rate; PTPoes per cycle, inspiratory oesophageal pressure

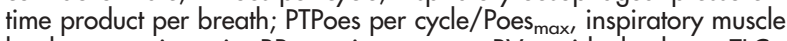
load to capacity ratio; RR, respiratory rate; RV, residual volume; TLC, total lung capacity; Te, expiratory time; Ti, inspiratory time; Ti/Ttot, duty cycle; Tlim, endurance time; $V_{E}$, minute ventilation; $\mathrm{VT}_{\mathrm{T}}$, tidal volume.
} 


\section{Pressure measurements}

An oesophageal balloon catheter (Ackrad Laboratories, NJ, USA) was positioned in the standard manner. ${ }^{20}$ Oesophageal pressure (Poes) was measured using a differential pressure transducer $\left( \pm 300 \mathrm{~cm} \mathrm{H}_{2} \mathrm{O}\right.$; Validyne, CA, USA) and amplified by a carrier amplifier (Validyne). All signals were sampled at $100 \mathrm{~Hz}$ and passed via an analogue digital board to a computer (Apple Computers, CA, USA) running Labview 4.1 software (National Instruments, TX, USA). Dynamic lung compliance (CLdyn) was calculated during resting breathing by dividing tidal volume (VT) by the oesophageal pressure difference at points of zero flow.

\section{Maximum inspiratory pressure}

Maximum inspiratory efforts were repeated from functional residual capacity (FRC) at least 10 times until three reproducible efforts were obtained. Each subject performed the manoeuvre using both a flange and tube mouthpiece in random order and the peak oesophageal pressure (Poes $\max$ ) with either mouthpiece was determined. Poes ${ }_{\max }$ was defined as the inspiratory muscle capacity.

\section{Inspiratory muscle endurance testing}

Inspiratory muscle endurance was measured using a negative pressure threshold loading technique developed in our laboratory, ${ }^{12}$ in which subjects breathe against a constant level low resistance inspiratory threshold loading device. ${ }^{21}$ Patients with CHF and healthy controls were exposed to a similar negative pressure $\left(40 \%\right.$ Poes $\left._{\max }\right)$ and underwent $2-5$ practice endurance runs, up to the target pressure, to become accustomed to the apparatus and the sensation of dyspnoea. During the first minute of the test the threshold pressure was progressively made more subatmospheric from $-10 \mathrm{~cm} \mathrm{H}_{2} \mathrm{O}$ to $40 \%$ Poes $_{\max }$. No restrictions were placed on the pattern of breathing and no instructions were given regarding the breathing pattern to adopt. Tlim was the period from when the negative pressure imposed reached $40 \%$ Poes $_{\max }$ to task failure. All participants continued until the level of dyspnoea was intolerable and task failure was defined as the inability to generate the target pressure and open the valve.

We also used data obtained from 35 normal subjects performing the same protocol at a more negative threshold pressure $\left(70 \%\right.$ Poes $\left._{\max }\right)$. The data from 30 of these subjects have been presented previously ${ }^{12}$ and these data were combined with additional data from five extra subjects (mean age 61 years).

\section{Inspiratory muscle load, inspiratory muscle contraction rate, and expiratory pressure}

Inspiratory oesophageal pressure-time product per breath cycle (PTPoes per cycle) was averaged for the whole test. PTPoes per cycle was defined as the inspiratory muscle load. ${ }^{12}$ Peak inspiratory oesophageal pressure normalised for the time to peak oesophageal pressure ( Poes $_{\text {peak }} /$ TTPoes $_{\text {peak }}$ ) was used as an index of inspiratory muscle contraction rate, whereas peak expiratory oesophageal pressure ( Poes $_{\exp }$ ) was used as a measure of intrapleural pressure change during expiration. As in previous studies, ${ }^{12}{ }^{13}$ the inspiratory muscle load to capacity ratio was calculated as PTPoes per cycle/ Poes $_{\max }$.

\section{Data analysis}

Our first comparison was between patients with CHF and healthy controls exposed to the same inspiratory load $(40 \%$ Poes $\left._{\max }\right)$. In line with our previous reports, ${ }^{12}{ }^{13}$ Tlim values were logarithmically converted (log Tlim) and plotted against the corresponding inspiratory muscle load to capacity ratios (PTPoes per cycle/Poes max $_{\text {) }}$, which allowed a second comparison to be made with control subjects studied at the higher threshold of $70 \%$ Poes $_{\max }$. This approach was used because none of the normal subjects experienced task failure at $40 \%$ Poes $_{\max }$ and because values of PTPoes per cycle/Poes max $_{\text {max }}$ observed in patients with CHF at $40 \%$ Poes $_{\max }$ were comparable to those obtained in normal subjects at $70 \%$ Poes $_{\max }$. To ensure that this comparison was appropriate and valid with respect to the age of the patients, modification of the previously published normal values ${ }^{12}$ was performed following additional studies in older subjects, as described above. Nevertheless, the additional normal data resulted in only a minor modification of the previously published values. ${ }^{12}$ The modified equation used in the current study to describe normal values was $\log$ Tlim $=3.5-3.6^{*}$ PTPoes per cycle/Poes $_{\max }(r=0.84, \mathrm{p}<0.0001)$.

Data are expressed as mean (SD) unless stated otherwise. Differences between patients with CHF and healthy controls were assessed using unpaired $t$ tests. Correlation between percentage predicted log Tlim and Poes peak $_{\text {TTPoes }}$ peak was assessed using simple linear regression analysis. A value of $\mathrm{p}<0.05$ was considered significant.

\section{RESULTS}

\section{Cardiac and pulmonary function}

All patients had severe left ventricular dysfunction (LVEF $22.5(4.0) \%$ and LVEDD $7.1(0.5) \mathrm{cm}$; table 1). Five patients had a New York Heart Association (NYHA) classification score of III and five had a score of II. Forced expiratory volume in 1 second $\left(\mathrm{FEV}_{1}\right)$, forced vital capacity (FVC), $\mathrm{FEV}_{\mathrm{l}} / \mathrm{FVC}$, total lung capacity (TLC), residual volume (RV), and FRC were similar in both groups (table 2).

\section{Inspiratory muscle strength, breathing pattern, dynamic lung compliance, and inspiratory muscle load to capacity ratio}

Poes $_{\max }$ tended to be lower in patients with CHF but this was not significantly reduced compared with the controls (table 3). Although patients with CHF and healthy controls were exposed to the same relative threshold load $(40 \%$ Poes $\left._{\max }\right)$, all CHF patients discontinued the test within 30 minutes (mean Tlim 306 s) whereas all healthy controls sustained the target negative pressure for this time, at which time the test was terminated $(\mathrm{p}<0.0001)$.

In both patients with $\mathrm{CHF}$ and controls the pattern of breathing established at the start varied little throughout the test. Patients with $\mathrm{CHF}$ had higher minute ventilation $(\mathrm{VE}$, $\mathrm{p}=0.05)$ and faster respiratory rate $(\mathrm{RR}, \mathrm{p}=0.05)$, with a shorter expiratory time $(\mathrm{Te}, \mathrm{p}=0.03)$ and longer duty cycle (Ti/Ttot, $p=0.001)$, but a similar inspiratory time (Ti, $\mathrm{p}=0.95$ ) compared with the healthy controls (table 3 ). Furthermore, there was a reduction in CLdyn in the CHF group $(p=0.006)$ accompanied by a slower Poes peak $/$ TTPoes $_{\text {peak }}(p=0.003)$ and higher Poes $\exp (p=0.004)$.

The net effect of these changes in breathing pattern in patients with CHF was to increase the inspiratory muscle load to capacity ratio (PTPoes per cycle/Poes max,$p=0.001$ ). Although the reduction in Cudyn would have contributed to the increased PTPoes per cycle/Poes max $_{\text {, we observed a }}$ positive correlation between PTPoes per cycle/Poes $\max$ and Ti/Tot $(r=0.84, \mathrm{p}=0.002)$ but not Cldyn $(\mathrm{p}=0.4)$. This, combined with the fact that the internal load as a percentage of the total load per breath calculated from Cudyn and mean Poes per cycle was approximately $18 \%$ in CHF patients and $11 \%$ in healthy controls, suggests that the major factor influencing the increase in PTPoes per cycle/Poes $\max$ in patients with CHF was prolongation of the duty cycle.

\section{Factors reducing endurance time}

By plotting log Tlim against PTPoes per cycle/Poes max $_{\text {, the }}$ inspiratory muscle endurance of patients with CHF was 
Table 1 Demographic and functional characteristics of patients with chronic heart failure

\begin{tabular}{clllllll}
\hline Patient no Sex & Age (years) & LVEF (\%) & LVEDD (cm) & NYHA & Aetiology & Medications \\
\hline 1 & F & 71 & 20 & 7.8 & III & DCM & Asp, Diu, Nit, Ca-a, ACEi, BB \\
2 & $M$ & 69 & 20 & 6.2 & DCM & Asp, Diu, Dig, ACEi, \\
3 & $M$ & 73 & 20 & 6.9 & II & DCM & War, Dig, Diu, ACEi \\
4 & $M$ & 53 & 25 & 7.1 & II & CAD & Asp, Diu (2), Nit, A-A, ACEi, \\
5 & $M$ & 51 & 25 & 6.7 & II & CAD & Asp, Diu, A-A, ACEi, BB \\
6 & $M$ & 35 & 15 & 8.0 & War, Diu (2), Dig, ACEi \\
7 & $M$ & 56 & 25 & 7.4 & III & CAD & War, Diu (2), ACEi, BB \\
8 & $M$ & 78 & 25 & 7.4 & III & CAD & Diu (2), Nit, ACEi, BB \\
9 & $M$ & 73 & 30 & 6.4 & III & CAD & War, Diu, Dig, Ca-a, ACEi \\
10 & $M$ & 67 & 20 & 7.1 & III & DCM & Asp, ACEi \\
\hline
\end{tabular}

$\mathrm{LVEF}=$ left ventricular ejection fraction; $\mathrm{LVEDD}=$ left ventricular end diastolic dimension; $\mathrm{NYHA}=$ New York Heart Association functional class; $\mathrm{DCM}=$ dilated cardiomyopathy; $C A D=$ coronary artery disease; $A s p=$ aspirin; Diu = diuretic; Nit= nitrate; $C a-a=$ calcium antagonist; $A C E i=a n g i o t e n s i n$ converting enzyme inhibitor; $B B=$ beta blocker; $D i g=$ digoxin; $W a r=$ warfarin; $A-A=$ antiarrhythmic.

directly compared with the modified regression equation for normal controls (fig 1). The mean log Tlim for the patients with CHF was 86.7 (8.9)\% predicted (range 69.8-96.0), with only three of the 10 patients having a value of less than $85 \%$ predicted (patients 1, 3 and 4; fig 1). Therefore, despite the fact that the CHF group had a substantial reduction in Tlim compared with controls set at a similar target negative pressure, Tlim was nearly appropriate when expressed as a function of the PTPoes per cycle/Poes max $_{\text {. Finally, direct }}$ correlation was observed between percentage predicted log

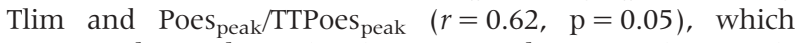
suggests that a slower inspiratory muscle contraction rate is observed during loaded breathing in patients with CHF.

\section{DISCUSSION}

In patients with moderate symptomatic heart failure, endurance time was greatly reduced primarily as a consequence of the breathing strategy adopted, which caused an increase in the inspiratory muscle load relative to the inspiratory muscle capacity. However, given this ventilatory pattern, most of the patients with heart failure sustained an endurance time that was nearly appropriate for the inspiratory muscle load to capacity ratio, suggesting that task failure is not due to poor endurance characteristics of the inspiratory muscles.

\section{Critique of method}

\section{Comparison with previous studies}

Two separate methods have previously been used to assess inspiratory muscle endurance in CHF. ${ }^{14}{ }^{15}$ Both studies showed an overall reduction in endurance, and both suggested that the likely cause was an increase in the inspiratory muscle load..$^{15}$ Mancini et al ${ }^{15}$ used an incremental isocapnic voluntary ventilation test. Despite this volume loading method being well tolerated and demonstrating a lower maximal sustainable ventilatory capacity in patients with CHF, this technique is limited when applied to CHF patients with evidence of airflow obstruction. Indeed, although lower in heart failure, when maximal sustainable ventilatory capacity was normalised for $\mathrm{FEV}_{1}$ there was little difference between patients with CHF and controls. To overcome the influences of respiratory frequency and underlying airways disease inherent in the volume loading tests, ${ }^{22}$ Walsh et $a l^{14}$ used an incremental pressure loading technique. $^{23}$ However, subsequent studies have questioned the usefulness of such incremental tests, as incremental loading tasks have a substantial learning effect ${ }^{24}$ and may reflect strength more than endurance. ${ }^{25}$ Incremental tests take no account of breathing strategy and therefore do not accurately reflect the load on the inspiratory muscles, a problem that is overcome if endurance time is expressed as a function of the inspiratory muscle load to capacity ratio. ${ }^{12}$

\section{Why set the threshold pressure level at $40 \%$ of maximal? \\ In our previous studies a threshold pressure of $70 \%$ Poes $_{\max }$ was used. ${ }^{12}{ }^{13}$ However, consistent with the study of Hammond et al, ${ }^{4}$ we observed that patients with CHF reached task failure prematurely at much lower target pressures. Accordingly, we determined from pilot studies that $40 \%$ Poes $_{\max }$ was the highest threshold pressure that was acceptable to the patients and allowed them to achieve an}

Table 2 Mean (SD) differences in demographic data and lung function tests between healthy controls $(\mathrm{HC})$ and patients with chronic heart failure (CHF)

\begin{tabular}{|c|c|c|c|c|}
\hline & $\mathrm{HC}$ & CHF & $p$ value & $\mathrm{HC}-\mathrm{CHF}(95 \% \mathrm{Cl})$ \\
\hline Age (years) & $54.0(20.1)$ & $62.6(13.4)$ & 0.3 & $-8.6(-24.7$ to 7.5$)$ \\
\hline $\mathrm{BMI}\left(\mathrm{kg} / \mathrm{m}^{2}\right)$ & $23.7(5.4)$ & $26.8(1.9)$ & 0.1 & $3.1(-0.8$ to 7.0$)$ \\
\hline $\mathrm{FEV}_{1}$ (l) & $3.7(1.1)$ & $2.7(0.7)$ & 0.1 & $0.9(-0.1$ to 2.0$)$ \\
\hline $\mathrm{FEV}_{1}(\%$ pred) & 107.8 (18.4) & $86.2(21.4)$ & & \\
\hline FVC (I) & $4.8(1.4)$ & $3.7(0.9)$ & 0.1 & $1.1(-0.3$ to 2.4$)$ \\
\hline FVC (\% pred) & 112.6 (19.1) & $91.2(18.9)$ & & \\
\hline $\mathrm{FEV}_{1} / \mathrm{FVC}$ & $76.7(7.5)$ & $74.8(9.7)$ & 0.7 & $1.9(-7.4$ to 11.1$)$ \\
\hline TLC (I) & $7.0(1.7)$ & $6.3(1.6)$ & 0.5 & $0.7(-1.3$ to 2.6$)$ \\
\hline TLC (\% pred) & $102.4(6.8)$ & $92.4(16.7)$ & & \\
\hline RV (I) & $2.0(0.2)$ & $2.3(0.8)$ & 0.3 & $-0.3(-1.0$ to 0.4$)$ \\
\hline RV (\% pred) & $99.1(18.1)$ & $97.7(22.4)$ & & \\
\hline FRC (I) & $3.6(1.0)$ & $3.3(1.1)$ & 0.7 & $0.2(-1.0$ to 1.5$)$ \\
\hline FRC ( $\%$ pred) & 105.1 (18.7) & $94.3(26.3)$ & & \\
\hline
\end{tabular}

$\mathrm{BMI}=$ body mass index; $\%$ pred $=$ percentage predicted $; \mathrm{FEV}_{1}=$ forced expiratory volume in 1 second; $F \mathrm{FC}=$ forced vital capacity; $\mathrm{TLC}=$ total lung capacity; $\mathrm{RV}=$ residual volume; $\mathrm{FRC}=$ functional residual capacity; $95 \% \mathrm{Cl}=95 \%$ confidence intervals.

$95 \%$ confidence intervals and $p$ values refer to the differences observed between the absolute values 
Table 3 Mean (SD) differences between healthy controls (HC) and patients with chronic heart failure (CHF) in inspiratory muscle strength, pulmonary mechanics, ventilatory breathing pattern, inspiratory muscle load, and endurance time during negative pressure threshold loading

\begin{tabular}{|c|c|c|c|c|}
\hline & $\mathrm{HC}$ & $\mathrm{CHF}$ & $p$ value & $\mathrm{HC}-\mathrm{CHF}(95 \% \mathrm{Cl})$ \\
\hline RR (breaths/min) & $15.5(6.4)$ & $20.5(3.6)$ & 0.05 & $-5.0(-9.9$ to -0.1$)$ \\
\hline VT (ml) & $640(287)$ & $574(164)$ & 0.5 & $65.6(-154$ to 285$)$ \\
\hline VE (I/min) & $8.8(3.0)$ & $11.4(2.7)$ & 0.05 & $-2.7(-5.4$ to -0.02$)$ \\
\hline $\mathrm{Ti}(\mathrm{s})$ & $1.9(0.8)$ & $1.9(0.4)$ & 0.95 & $0.02(-0.60$ to 0.63$)$ \\
\hline $\mathrm{Te}(\mathrm{s})$ & $3.0(5.8)$ & $1.1(0.3)$ & 0.03 & $1.9(0.3$ to 3.5$)$ \\
\hline $\mathrm{Ti} /$ Ttot & $0.43(0.14)$ & $0.62(0.07)$ & 0.001 & $-0.2(-0.3$ to -0.1$)$ \\
\hline Cidyn $\left(\mathrm{ml} / \mathrm{cm} \mathrm{H} \mathrm{H}_{2} \mathrm{O}\right)$ & $157(43)$ & $98(42)$ & 0.006 & 59.1 (19.2 to 99.0$)$ \\
\hline Poes $_{\max }\left(\mathrm{cm} \mathrm{H}_{2} \mathrm{O}\right)$ & $-117.7(23.6)$ & $-100.0(18.3)$ & 0.1 & $-17.6(-37.5$ to 2.2$)$ \\
\hline $\operatorname{Tlim}(s)$ & $1800^{*}$ & $306(190)$ & $<0.0001$ & 1494 (1368 to 1620$)$ \\
\hline Mean Poes/breath $\left(\mathrm{cm} \mathrm{H}_{2} \mathrm{O}\right)$ & $-37.8(10.2)$ & $-32.9(7.3)$ & 0.24 & $-4.9(-13.2$ to 3.5$)$ \\
\hline PTPoes per cycle/Poes $\max$ & $0.13(0.05)$ & $0.21(0.04)$ & 0.001 & $-0.17(-0.11$ to -0.03$)$ \\
\hline Poes $_{\text {peak }} / T_{T P o e s}$ peak $\left(\mathrm{cm} \mathrm{H}_{2} \mathrm{O} / \mathrm{s}\right)$ & $-95.5(35.2)$ & $-53.5(19.9)$ & 0.004 & $-42.0(-69.0$ to -14.9$)$ \\
\hline $\mathrm{Poes}_{\exp }\left(\mathrm{cm} \mathrm{H} \mathrm{H}_{2} \mathrm{O}\right)$ & $-1.9(2.7)$ & $1.7(2.1)$ & 0.004 & $-3.6(-5.8$ to -1.3$)$ \\
\hline
\end{tabular}

$\mathrm{RR}=$ respiratory rate; $\mathrm{VT}=$ tidal volume; $\mathrm{VE}=$ minute ventilation; $\mathrm{Ti}=$ inspiratory time; $\mathrm{Te}=$ expiratory time; $\mathrm{Ti} /$

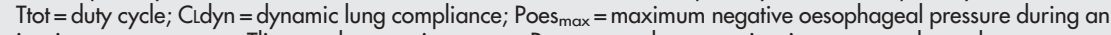
inspiratory manoeuvre; Tlim = endurance time; mean Poes per cycle = mean inspiratory oesophageal pressure per breath; PTPoes per cycle = inspiratory oesophageal pressure time product per breath; PTPoes per cycle/

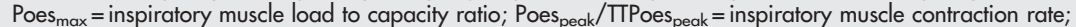

Poes $_{\text {exp }}=$ peak expiratory oesophageal pressure; $95 \% \mathrm{Cl}=95 \%$ confidence intervals.

*All age matched controls were instructed to stop the test at 30 minutes (1800 s)

endurance time of a sufficient duration to assess inspiratory muscle endurance satisfactorily. Furthermore, as the PTPoes per cycle/Poes $\max _{\max }$ values observed in patients with CHF at the $40 \%$ Poes $_{\max }$ threshold level were numerically comparable to those observed in normal subjects exposed to a $70 \%$ Poes $_{\max }$ threshold level, additional comparisons could be made between CHF patients and normal subjects (fig l).

\section{Significance of findings}

Factors influencing Tlim

A higher PTPoes per cycle/Poes ${ }_{\max }$ has been shown to be associated with a reduced Tlim in normal subjects. ${ }^{12}$ In the current study a different breathing strategy was adopted in CHF patients, reflected as an increased RR, reduced Te, and therefore increased Ti/Ttot with little difference in Ti. Consistent with this trend, during exercise heart failure is characterised by an increase in $\mathrm{VE}, \mathrm{RR}^{26-28}$ and $\mathrm{Ti} / \mathrm{Tt}$ tot. ${ }^{29}$ Although preservation of $\mathrm{Ti}$ in patients with heart failure allows an adequate Vт to be maintained and gas exchange to occur, this is at a cost since, to accommodate the increase in

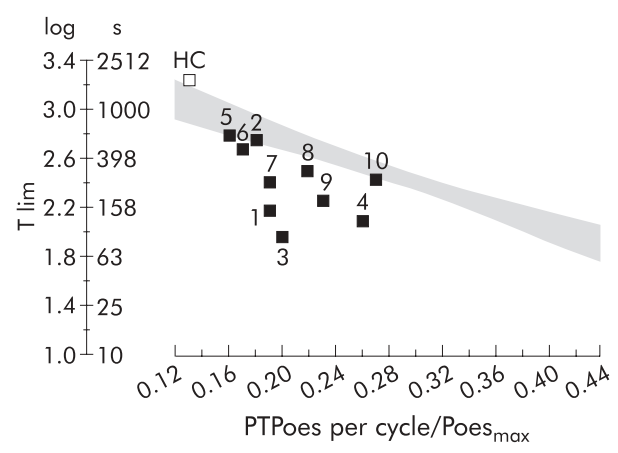

Figure 1 Plot of endurance time (log Tlim and Tlim) against the inspiratory muscle load to capacity ratio (PTPoes per cycle/Poes max $_{\text {) }}$ in 10 patients with chronic heart failure. The shaded area represents the regression equation and $95 \%$ confidence bands, which has been modified from previous published data. ${ }^{12}$ Although the healthy controls $(\mathrm{HC})$ exposed to $40 \%$ Poes $_{\max }$ do not fit the regression equation as these subjects did not reach task failure, the mean value for this group has been added for comparison. Patients 2, 5, 6, 7, 8, 9 and 10 had a log Tlim of $\geqslant 85 \%$ predicted.
VE, RR increases and Te falls resulting in increased Ti/Ttot. This adversely impacts on muscle energetics by reducing the time available for recovery before the onset of another inspiratory contraction. ${ }^{30}$ Thus, in the present study, Tlim was reduced in CHF patients as a consequence of the increased inspiratory muscle load to capacity ratio.

This increase in Ti/Ttot may be an indirect result of a reduction in Cudyn. Similar to previous studies, ${ }^{31}$ lung compliance was decreased in patients with CHF compared with controls, and although this degree of reduction in lung compliance only accounts for part of the higher inspiratory muscle load to capacity ratio observed in patients with heart failure, the increased respiratory system impedance associated with the reduction in lung compliance would increase the central inspiratory motor output and modify breathing strategy. ${ }^{33}$ In fact, the mechanical constraints imposed by lung stiffness in CHF may necessitate the preferential increase in RR rather than VT. These data suggest that the high intrinsic load in CHF contributed, in part, directly to the increase in the inspiratory muscle load, but the indirect effects on breathing pattern had a greater influence on the magnitude of the inspiratory muscle load. Since this breathing pattern resulted in a reduction in Tlim in CHF patients compared with healthy controls exposed to the same relative load, it could be considered as a maladaptive ventilatory response to high inspiratory loads.

\section{Inspiratory muscle endurance in $\mathrm{CHF}$}

Although the patients with CHF had a reduction in Tlim compared with healthy controls exposed to a similar inspiratory negative pressure, compared with healthy controls with similar PTPoes per cycle/Poes $\max$ values the CHF patients had Tlim values that were largely appropriate for the inspiratory muscle load to capacity ratio, indicating preservation of the endurance properties of the inspiratory muscles. In addition, a slower inspiratory muscle contraction rate was observed in patients with CHF which may be a reflection of the fact that the diaphragm of patients with heart failure has increased numbers of slow type I endurance fibres and decreased fast type II strength fibres. ${ }^{34}$ The relevance of these findings in vivo is supported by data from our laboratory showing a shift to the left of the force-frequency curve in the diaphragm, an adaptation also consistent with a relative increase in endurance fibres. ${ }^{9}$ 
The effect on cardiac function of decreasing pleural pressures during inspiratory manoeuvres and of positive pleural pressures during ventilatory assistance is well documented. ${ }^{35-40}$ A more negative (subatmospheric) pressure during inspiration causes an increase in left ventricular afterload by increasing the transmural pressure across the left ventricular wall, and consequently results in a fall in the left ventricular output. Although we did not measure central haemodynamic responses, inspiratory threshold loading would be expected to cause a reduction in cardiac output and have a deleterious effect on inspiratory muscle function. $^{41}{ }^{42}$ However, in the current study CHF patients developed a breathing strategy that counterbalanced the adverse effect of large inspiratory negative pleural pressure on cardiac output by generating a more positive pleural pressure in expiration compared with the control subjects (Poes $_{\text {exp }}$ was subatmospheric in the controls), which would reduce afterload and increase cardiac output -an effect that would preserve inspiratory muscle function. Although a positive pressure in expiration can be a consequence of either increased abdominal muscle activity or an indicator of dynamic hyperinflation, the patients with CHF had an $\mathrm{FEV}_{\mathrm{l}} / \mathrm{FVC}$ ratio similar to the healthy controls and we

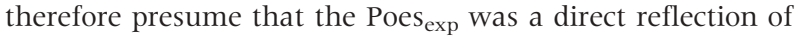
compensatory expiratory muscle activity. Nevertheless, this positive impact on cardiac output would be offset by the negative effect on muscle energetics due to the forced expiratory phase causing a reduction in Te and therefore a decrease in inspiratory muscle rest before the next inspiratory contraction.

\section{Clinical significance}

Previous data ${ }^{14}$ have shown that inspiratory muscle endurance is reduced in heart failure and is a possible cause of reduced exercise performance. Some studies have suggested an increase in inspiratory muscle load relative to inspiratory muscle capacity as a cause of dyspnoea and exercise intolerance, ${ }^{10}$ while other studies have reached contrasting conclusions. ${ }^{43}$ The findings of the current study indicate that, although endurance time is greatly reduced in heart failure, this is because patients with CHF adopt a maladaptive breathing strategy with a prolonged Ti/Ttot. These data support the clinical findings of Bernardi $e t$ a $l^{44}$ that respiratory pattern training techniques, which aim to modify the maladaptive breathing pattern and impose a more physiologically effective breathing strategy, alleviate dyspnoea and improve exercise tolerance in patients with CHF. Furthermore, this approach is consistent with our observation in normal subjects that changing breathing strategy can alter Tlim but without modifying inspiratory muscle endurance, when Tlim is expressed as a function of PTPoes per cycle/Poes max $^{12}$

In conclusion, endurance time is significantly shorter in patients with CHF due to the breathing pattern adopted, which results in an increase in the inspiratory muscle load relative to the inspiratory muscle capacity. However, when inspiratory muscle endurance is expressed as a function of endurance time and the inspiratory muscle load to capacity ratio, the endurance properties of the inspiratory muscle are relatively well preserved in CHF. Maladaptive breathing strategies resulting in an increase in inspiratory muscle load are the principal contributors to task failure during loaded breathing in CHF rather than the strength or endurance of the inspiratory muscle pump.

\footnotetext{
Authors' affiliations

N Hart, N B Pride, M Green, M I Polkey, Respiratory Muscle Laboratory, Royal Brompton Hospital, London, UK

M T Kearney, A M Shah, Department of Cardiology, Guy's, King's and
}

St Thomas' School of Medicine King's College Hospital, London, UK F Lofaso, Department of Clinical Physiology, Raymond Poincaré Hospital, Garches, France

J Moxham, Department of Respiratory Medicine \& Allergy, Guy's, King's and St Thomas' School of Medicine, King's College Hospital, London, UK

$\mathrm{NH}$ was funded by the Dorothy Osbourne Legacy (Royal Brompton Hospital Trust Fund). MIP's research group receives support from the British Heart Foundation.

\section{REFERENCES}

1 Lipkin DP, Poole-Wilson PA. Symptoms limiting exercise in chronic heart failure. BMJ 1986;292:1030-1.

2 Weber KT, Szidon JP. Exertional dyspnoea. In: Weber KT, Janicki JS, eds. Cardiopulmonary exercise testing. Philadelphia: WB Saunders, 1986:290-301.

3 Killian KJ, Jones N. Respiratory muscles and dyspnea. Clin Chest Med 1988;9:237-48.

4 Hammond K, Baver K, Sharp J, et al. Respiratory muscle strength in congestive heart failure. Chest 1990;98:1091-4.

5 McParland C, Khrishnan B, Wang Y, et al. Inspiratory muscle weakness and dyspnoea in chronic heart failure. Am Rev Respir Dis 1992;146:467-72.

6 Ambrosino N, Opasich C, Crotti P, et al. Breathing pattern, ventilatory drive, and respiratory muscle strength in patients with chronic heart failure. Eur Respir J 1994;7:17-22.

7 Mancini DM, Henson D, LaManca J, et al. Respiratory muscle function and dyspnea in patients with chronic congestive heart failure. Circulation 1992:86:909-18

8 Evans SA, Watson L, Hawkins M, et al. Respiratory muscle strength in chronic heart failure. Thorax 1995;50:625-8.

9 Hughes PD, Polkey MI, Harris ML, et al. Diaphragm strength in chronic heart failure. Am J Respir Crit Care Med 1999;160:529-34.

10 Hughes PD, Hart N, Hamnegard CH, et al. Inspiratory muscle relaxation rate slows during exhaustive treadmill walking in patients with chronic heart failure. Am J Respir Crit Care Med 2001;163:1400-3.

11 Kufel TJ, Pineda RG, Junega R, et al. Diaphragm function after intense exercise in congestive heart failure patients. Eur Respir J 2002;20:1399-405.

12 Hart N, Hawkins $\mathrm{P}$, Hamnegard $\mathrm{C}-\mathrm{H}$, et al. A novel clinical test of respiratory muscle endurance. Eur Respir J 2002;19:232-9.

13 Hart N, Nickol AH, Cramer D, et al. The effect of isolated unilateral and bilateral diaphragm weakness on exercise performance. Am J Respir Crit Care Med 2002; 165:1265-70.

14 Walsh JT, Andrews R, Johnson P, et al. Inspiratory muscle endurance in patients with chronic heart failure. Heart 1996;76:332-6.

15 Mancini DM, Henson D, LaManca J, et al. Evidence of reduced respiratory muscle endurance in patients with heart failure. J Am Coll Cardiol 1994;24:972-81.

16 Clanton TL. A breakthrough in the functional evaluation of the inspiratory muscle pump. Eur Respir J 2002;19:207-8.

17 Poole-Wilson PA. Future perspectives in the management of congestive heart failure. Am J Cardiol 1990;66:462-7.

18 British Thoracic Society. Guidelines for the measurement of respiratory function. Respir Med 1994;88:165-94.

19 Quanjer PH. Standardized lung function testing. Eur Respir J 1993;6(Suppl 16):5-30s.

20 Baydur A, Behrakis PK, Zin WA, et al. A simple method for assessing the validity of the esophageal balloon technique. Am Rev Respir Dis 1982; 126:788-91.

21 Chen RC, Que CL, Yan S. Introduction to a new inspiratory threshold loading device. Eur Respir J 1998;12:208-11.

22 Nickerson BG, Keens TG. Measuring ventilatory muscle endurance in humans as sustainable inspiratory pressure. J Appl Physiol 1982;52:768-72.

23 Martyn JB, Moreno RH, Pare PD, et al. Measurement of inspiratory muscle performance with incremental threshold loading. Am Rev Respir Dis 1987; 135:919-23.

24 Eastwood PR, Hillman DR, Morton AR, et al. The effects of learning on the ventilatory responses to inspiratory threshold loading. Am J Respir Crit Care Med 1998; 158:1190-6.

25 Eastwood PR, Hillman DR, Finucane KE. Ventilatory responses to inspiratory threshold loading and role of muscle fatigue in task failure. J Appl Physiol 1994;76: 185-95.

26 Rubin SA, Brown HV. Ventilation and gas exchange during exercise in severe chronic heart failure. Am Rev Respir Dis 1984;129:S63-4.

27 Sullivan MJ, Higginbotham MB, Cobb FR. Increased exercise ventilation in patients with chronic heart failure: intact ventilatory control despite hemodynamic and pulmonary abnormalities. Circulation 1988;77:552-9.

28 Buller NP, Poole-Wilson PA. Mechanism of the increased ventiltory response to exercise in patients with chronic heart failure. Br Heart J 1990;63:281-3.

29 Agostoni P, Pellegrino R, Conca C, et al. Exercise hyperpnea in chronic heart failure: relationships to lung stiffness and expiratory flow limitation. $J$ Appl Physiol 2002;92:1409-16.

30 Bellemare F, Grassino A. Effect of pressure and timing of contraction on human diaphragm fatigue. J Appl Physiol 1982;53:1 190-5.

31 Christie R, Meakins J. The intrapleural pressure in congestive heart failure and its clinical significance. J Clin Invest 1934;13:323-45. 
32 Evans SA, Watson L, Cowley AJ, et al. Static lung compliance in chronic heart failure: relation with dyspnoea and exercise capacity. Thorax 1995; 50:245-8.

33 Killian KJ, Jones NL. The use of exercise testing and other methods in the investigation of dyspnea. Clin Chest Med 1984:5:99-108.

34 Tikunov B, Levine S, Mancini D. Chronic congestive heart failure elicits adaptations of endurance exercise in diaphragmatic muscle. Circulation 1997;95:910-6.

35 Chadda K, Annane D, Hart N, et al. Cardiac and respiratory effects of continuous positive airway pressure and non invasive ventilation in acute cardiac pulmonary oedema. Crit Care Med 2002;30:2457-61.

36 Bradley TD, Hall MJ, Ando S-I, et al. Hemodynamic effects of simulated obstructive apneas in humans with and without heart failure. Chest 2001;119:1827-35

37 Buda AJ, Pinsky MR, Ingels NB, et al. Effects of intrathoracic pressure on left ventricular performance. N Engl J Med 1979;300:453-9.

38 Hall MJ, Ando S-I, Floras JS, et al. Magnitude and time course of hemodynamic responses to Mueller maneuvres in patients with congestive heart failure. J Appl Physiol 1998;85:1476-84.
39 Karam M, Wise RA, Natarajan MS, et al. Mechanisms of decreased left ventricular stroke volume during inspiration in man. Circulation 1984;69:866-73

40 Naughton MT, Rahman A, Hara K, et al. Effects of continuous positive airway pressure on intrathoracic and left ventricular transmural pressures in patients with congestive heart failure. Circulation in patients with con $1995 ; 91: 1725-31$.

41 Aubier $M$, Trippenbach T, Roussos C. Respiratory muscle fatigue during cardiogenic shock. J Appl Physiol 1981;51:499-508.

42 Mancini D, Nazzaro D, Ferraro N, et al. Demonstration of respiratory muscle deoxygenation during exercise in patients with heart failure. J Am Coll Cardiol $1991 ; 18: 492-8$.

43 Mancini DM, La Manca J, Donchez L, et al. The sensation of dyspnea during exercise is not determined by the work of breathing in patients with heart failure. J Am Coll Cardiol 1996;28:391-5.

44 Bernardi L, Spadacini G, Bellwon J, et al. Effect of breathing rate on oxygen saturation and exercise performance in chronic heart failure. Lancet 1998;351:1308-11.

\section{LUNG ALERT}

\section{Systemic bioactivity of inhaled fluticasone is not affected by the presence of} emphysema

$\Delta$ Lipworth BJ, Lee DKC. The presence of emphysema does not affect the systemic bioactivity of inhaled fluticasone in severe chronic obstructive pulmonary disease. Br J Clin Pharmacol 2004;57:388-92.

nhaled corticosteroids reduce exacerbation frequency in COPD but it is not known whether the presence of emphysema, with decreased alveolar volume, results in impaired bioactivity. The systemic effects of fluticasone $2000 \mu \mathrm{g}$ daily were compared in patients with COPD who did and did not have emphysema (COPDE and COPD, respectively, both $\mathrm{n}=10$ ). The two groups had comparable airflow obstruction. The presence of emphysema was defined by carbon monoxide transfer factor $<60 \%$ in conjunction with appropriate spirometric, clinical, and radiological findings. Systemic bioactivity was assessed using overnight 10 hour urinary cortisol excretion corrected for creatinine (OUCC) as a marker of adrenal suppression and serum osteocalcin as an indicator of bone metabolism. OUCC and osteocalcin were measured at baseline and after 2 weeks of fluticasone.

OUCC values did not differ significantly between the COPD and COPDE groups before or after starting treatment with fluticasone, but in both groups fluticasone resulted in significant suppression of OUCC. Similarly, the serum osteocalcin concentration did not differ significantly between the two groups before or after starting treatment with fluticasone, but osteocalcin was significantly suppressed following fluticasone.

The results suggest that the degree of cortisol and osteocalcin suppression is similar in COPD patients with or without emphysema. The authors hypothesise that the site of absorption of fluticasone may therefore be more proximal than the alveoli. Patients with COPD on high dose fluticasone are susceptible to systemic adverse effects whether or not they have a significant component of emphysema.

A Jain

Vallabh Bhai Patel Chest Institute, Delhi, India; Aj1974@rediffmail.com 